\title{
C-Band Telemetry of Insect Pollinators Using a Miniature Transmitter and a Self-Piloted Drone
}

Shearwood, Jake; Aldabashi, Nawaf; Eltokhy, Amira; Franklin, Elizabeth; Raine, Nigel; Zhang, Chaochun; Palmer, Edward ; Cross, Paul; Palego, Cristiano

\section{IEEE Transactions on Microwave Theory and Techniques}

DOI:

https://doi.org/10.1109/TMTT.2020.3034323

Published: 01/01/2021

Peer reviewed version

Cyswllt i'r cyhoeddiad / Link to publication

Dyfyniad o'r fersiwn a gyhoeddwyd / Citation for published version (APA):

Shearwood, J., Aldabashi, N., Eltokhy, A., Franklin, E., Raine, N., Zhang, C., Palmer, E., Cross, P., \& Palego, C. (2021). C-Band Telemetry of Insect Pollinators Using a Miniature Transmitter and a Self-Piloted Drone. IEEE Transactions on Microwave Theory and Techniques, 69(1), 938946. https://doi.org/10.1109/TMTT.2020.3034323

\footnotetext{
Hawliau Cyffredinol / General rights

Copyright and moral rights for the publications made accessible in the public portal are retained by the authors and/or other copyright owners and it is a condition of accessing publications that users recognise and abide by the legal requirements associated with these rights.

- Users may download and print one copy of any publication from the public portal for the purpose of private study or research.

- You may not further distribute the material or use it for any profit-making activity or commercial gain

- You may freely distribute the URL identifying the publication in the public portal?
}

Take down policy

If you believe that this document breaches copyright please contact us providing details, and we will remove access to the work immediately and investigate your claim. 


\title{
C-Band Telemetry of Insect Pollinators Using a Miniature Transmitter and a Self-Piloted Drone
}

\author{
Jake Shearwood, Student Member, IEEE, Nawaf Aldabashi, Amira Eltokhy, Elizabeth L. Franklin, \\ Nigel E. Raine, Chaochun Zhang, Edward Palmer, Paul Cross, and Cristiano Palego, Member, IEEE
}

\begin{abstract}
A battery-less $5.8 \mathrm{GHz}$ transmitter, compact and lightweight enough to enable flight of $\sim 90 \mathrm{mg}$ honeybees and free roaming of bumblebees within their nest, was developed. The transmitter was coupled to a compact phased array antenna receiver to achieve angle of arrival (AOA) estimation and bee localization through a received signal strength indicator approach (RSSI). The receiver was integrated into a commercial Unmanned Aerial Vehicle (UAV) to support autonomous position updates based on the AOA estimates and a software development kit. A simple model predicting the system detection range was finally developed and is herein discussed. Our experimental results provide proof of concept towards autonomous tracking of tagged bees both in open air and in polytunnel settings.
\end{abstract}

Index Terms -Energy harvesting, localization, phased arrays, received signal strength indicator, telemetry, unmanned vehicle.

\section{INTRODUCTION}

$\mathrm{T}$ HE increasing rate of anthropogenic driven land-use change is presenting substantial challenges to pollinator communities and their associated plants [1]. Knowledge of how insects use space is critical to our ability to optimise agricultural and urban environments for pollinators such as bees [2]-[6].

Such knowledge is increasingly urgent in food production systems such as polytunnels, where bee foraging decisions and fruit yields can be optimised through the appropriate configuration of polytunnels within which they fly [5]. Current harmonic radar tracking equipment, the current gold standard for monitoring individual insect movements in space, allows only glimpses of their spatial behaviours. It is constrained by the restricted range; the fact that individuals can only be tracked one at a time; the need for a direct line of sight between the tagged bee and the radar receiver; and the need to remove the tag after each flight [6].

Herein we describe a ground-breaking technological advance

Manuscript received July 24, 2020; revised August 31, 2020; accepted September 20, 2020. Date of publication October 12, 2020; date of current version October 12, 2020. This work was supported by the Knowledge Economy Skills Scholarships (KESS 2, Ref: BUK226), the Agri-tech in China: Newton Network+ (Ref. SM024), the Natural Sciences and Engineering Research Council Discovery grant (2015-06783) and The W. Garfield Weston Foundation. This paper is an expanded version from the IEEE MTTS International Microwave Symposium, Los Angeles, CA, 2020.

J. Shearwood, N. Aldabashi, P. Cross and C. Palego are with Bangor University, Bangor, LL57 1UT UK (e-mail: c.palego@ bangor.uk). A. Eltokhy, was with Bangor University, Bangor, LL571UT UK. She is now with Rapid Bio-Labs, Talinn, 11314 Estonia (e-mail: a.eltokhy@ rapidbiolabs.com).

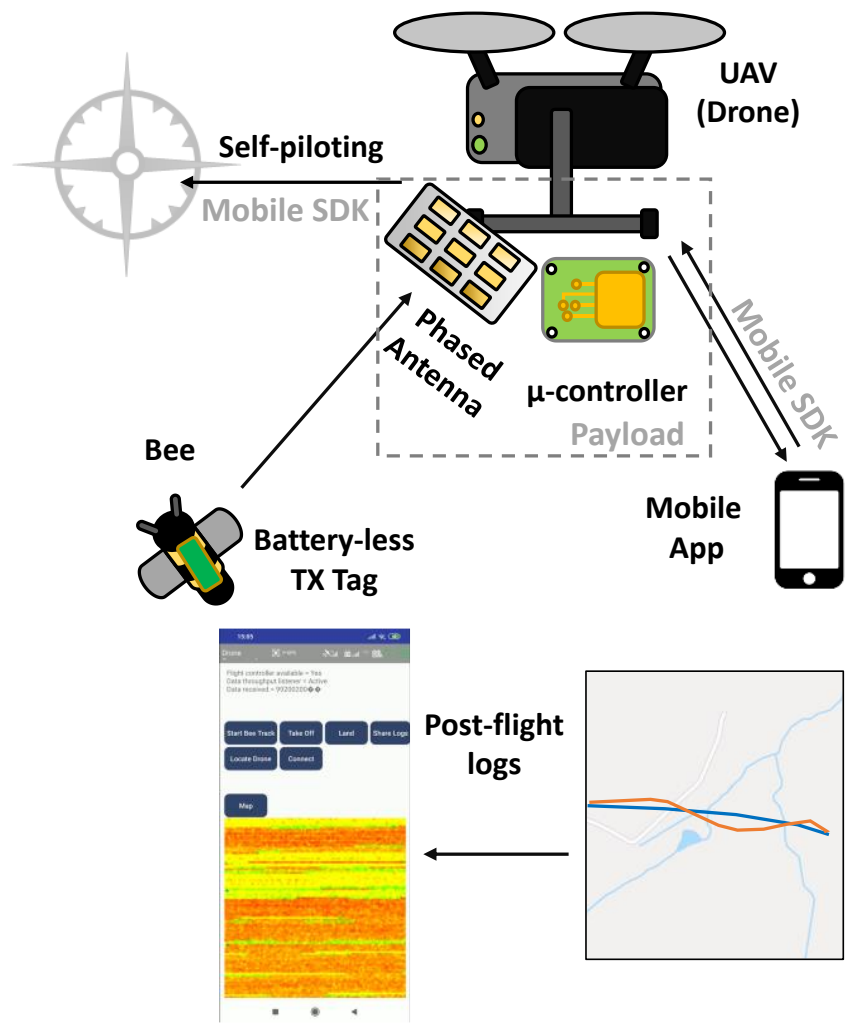

Fig. 1. Functional blocks of the present telemetry system based on a batteryless transmitter (TX) tag, a compact AOA detector and a self-piloted unmanned aerial vehicle (a commercial drone).

in insect telemetry designed to answer multiple fundamental questions about how pollinators (particularly bees) operate in space. The system portrayed in Fig. 1 comprises a miniaturised energy harvester to power a $5.8 \mathrm{GHz}$ bee-wearable transmitter; a phased-array antenna combined with a logarithmic detector and processing unit to compute the angle of arrival (AOA) and a WiFi module to support autonomous logging for Low Range

N. Raine is with the University of Guelph, Guelph ON, N1G 2W1, Canada (e-mail: nraine@uoguelph.ca).

E. Franklin was with the University of Guelph, Guelph ON, N1G 2W1, Canada. She is now with the Cornwall College Newquay, Newquay TR7 2LZ, UK (e-mail: elizabeth.bates@ cornwall.ac.uk).

Chaochun Zhang is with the China Agricultural University, Haidian District, Beijing, China (e-mail: Zhangcc@ cau.edu.cn).

Edward Palmer is with S\&A Produce UK Ltd, Hereford, HR1 3ET, UK (email: edward.palmer@sagroup.co.uk). 


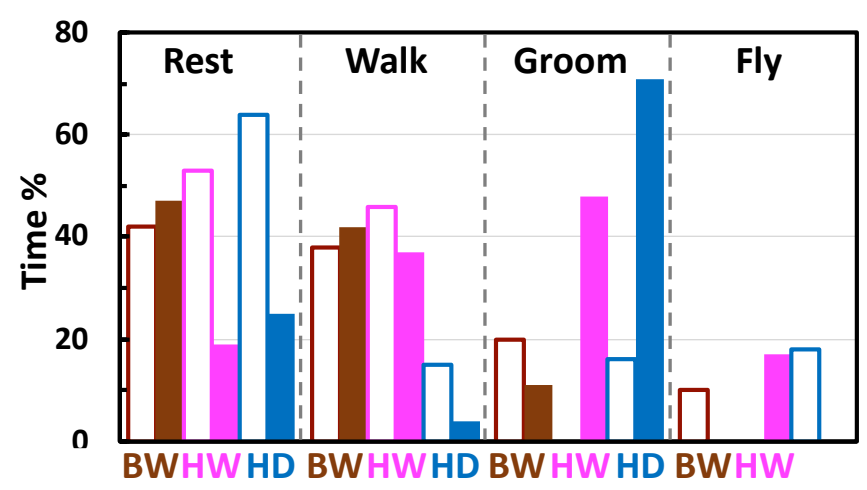

(a)

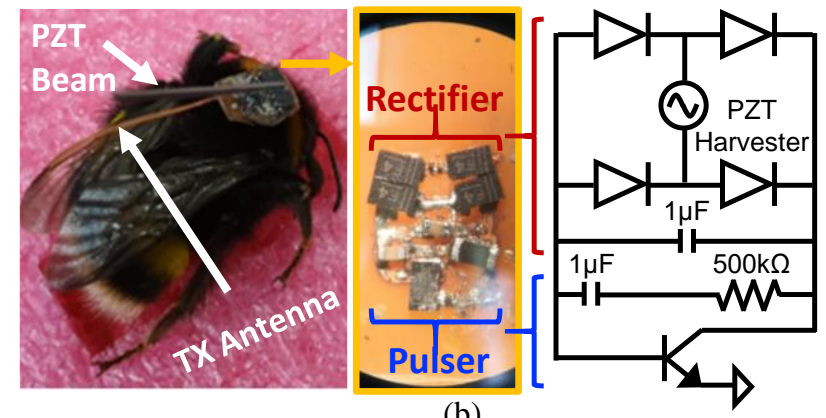

(b)

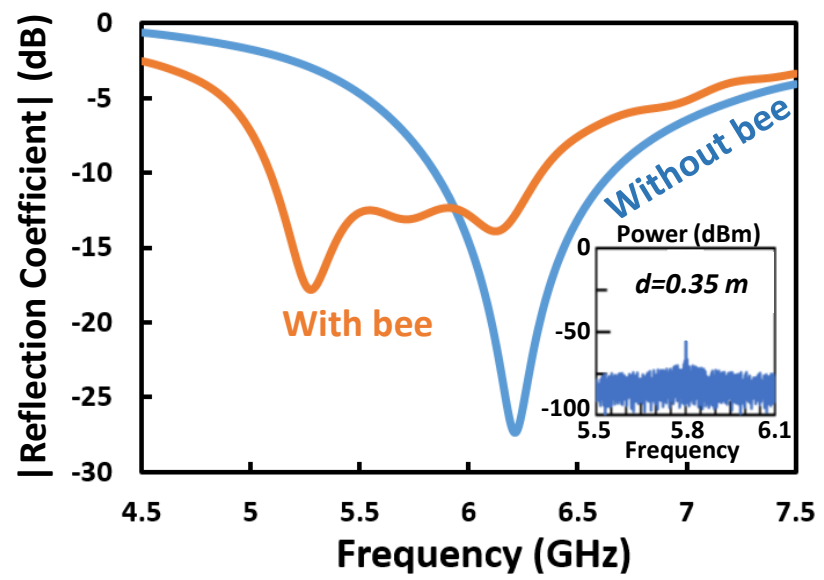

(c)

Fig. 2. (a) Percentage of time spent by bumblebees (BW), honeybee workers (HW) and honeybee drones (HD) in various activities by untagged control (open bars) and insects tagged with $80 \mathrm{mg}$ tag (filled bars). (b) Transmitter tag attached to a dorsal thorax of a bumblebee (Bombus terrestris) worker (left panel) with magnified power conditioning unit (right panel). (c) Transmit antenna reflection coefficient with and without loading effect from bee body. Inset: received signal spectrum $0.35 \mathrm{~m}$ away from the tag driven by a shaker.

sensor networks. A housing integrates the tracking system to an unmanned aerial vehicle, which is programmed to locate and autonomously follow the emitted signal.

\section{BATTERY-LESS TRANSMITTER TAG DESIGN}

Since weight tolerance varies widely between species, castes and environmental condition, a preliminary test surveyed different insect response when tagged with 3D printed mock tags of various weights. A cohort of 39 insects including approximately equal numbers of worker bumblebees (BW: Bombus impatiens), worker and drone honeybees (HW and HD: A. mellifera) were divided in treated (tagged) versus control (only paint marked) groups. BW and HW were wild caught and the HD were provided by the Guelph Honey Bee Research Center. A $~ 80 \mathrm{mg}$ tag matching the one in previous research [7] was used. The observed groups were placed in a $2.1 \times 1.2 \mathrm{~m}^{2}$ flight cage. After a 10 minute recovery period the subjects were recorded for four different activities (resting, walking, grooming and flying) during a 10 minute monitoring window.

The records are gathered for control (empty bars) and bees treated with the $80 \mathrm{mg}$ tag (solid bars) in Fig. 2(a). The tests outlined a scarce propension to flight, even in untagged insects and an increase in grooming time for honeybees. Later tests confirmed that while the $80 \mathrm{mg}$ tag could be carried by large insect such as carpenter bees (Xylocopa spp. $\sim 240 \mathrm{mg}$ ) and bumblebees ( $200 \mathrm{mg}$ ), it mostly inhibited honeybee flight [8].

By contrast, A $35 \mathrm{mg}$ tag corresponding to approximately a third of A. mellifera's mass ( $\sim 95 \mathrm{mg}$ ) enabled $90 \%$ of the tagged honeybees to fly. $4 \mathrm{~mm} \times 3 \mathrm{~mm}^{2}$ tags as lightweight as $30 \mathrm{mg}$ could be successfully fabricated on flexible $50 \mu \mathrm{m}$ FR4 substrate. However, their small footprint and challenging connections resulted in low tag yield. As such, the $1^{\text {st }}$ generation $80 \mathrm{mg}$ tags [7] were more consistently used on worker bumblebees in the experiments herein presented.

\section{A. Vibration energy harvester}

The electro-mechanical design of the piezoelectric harvester has been discussed elsewhere [7] and based on [9]. Briefly, as bee's wing-beat frequency varies depending on individual and ambient [10] a non-resonant harvester design was pursued with direct excitation from the insect's thoracic vibration.

The optimised piezoelectric beam dimensions were a tradeoff between mechanical handiness (cutting resolution, attachment surface) and the necessity to minimise flight impairment. It was found that a $16 \times 02 \times 0.38 \mathrm{~mm}^{3}$ beam resulted in a $3.6 \mu \mathrm{W}$ harvested power which approached the [7] model $3.7 \mu \mathrm{W}$ prediction when the 31 piezoelectric mode was considered. This is approximately $50 \%$ of the power that was harvested in [9] using (larger) beetles. A bimorph piezoelectric material [11] was used for this study. The full-wave rectifier displayed in Fig. 2(b) was based on Schottky diodes [12] a $1 \mu \mathrm{F}$ capacitor and a $500 \mathrm{k} \Omega$ resistive load to send a $1.5 \mathrm{~V}$ pulse per second with a $50 \%$ duty cycle. The scan time was set to match the pulse width of the emitting tag ( 0.5 seconds) so that multiple bee bearings were not received in a single scan. The received signal spectrum at a $0.35 \mathrm{~m}$ distance when the tag was driven by a mechanical shaker is shown in the inset of Fig. 2(c).

\section{B. Transmitter Antenna Design}

The antenna was designed to be as small, lightweight and isotropic as possible in order to 1) reduce physical hindrance to the bee; 2) minimise transmitter circuitry complexity to cope with continuous changes of direction during flight. The possibility of integrating the antenna and energy harvester was explored for weight minimisation but ruled out since the high dielectric constant of the energy harvester leads to low antenna efficiency and very low bandwidth. A simple monopole configuration with a thin $(0.28 \mathrm{~mm})$ wire was sufficiently omnidirectional for the application and only added $7 \mathrm{mg}$ ( $9 \div 20 \%$ depending on tag generation) to the tag weight. 


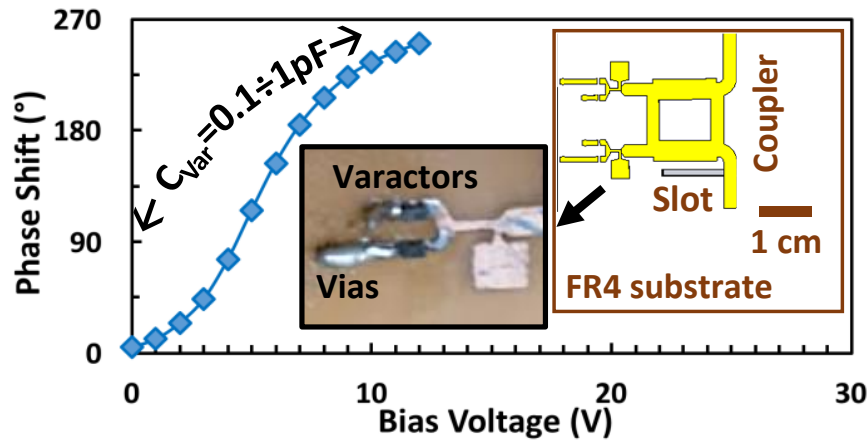

(a)

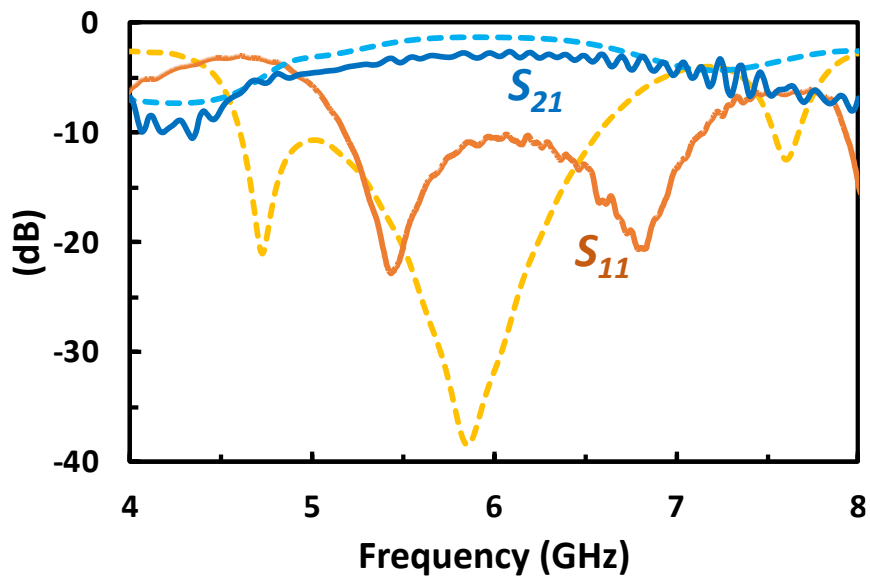

(b)

Fig. 3. (a) Phase shift vs applied bias voltage with load capacitance as a parameter. (b) Measured and simulated scattering parameters for the phase shifter.

The simulated impedance match of the transmitting antenna is shown in Fig. 2(c). The antenna was loaded through the (high dielectric constant and tangent loss) bee's body proximity. Hence $5.8 \mathrm{GHz}$ operation was obtained by designing the unloaded antenna to resonate at $6.2 \mathrm{GHz}$.

\section{RECEIVER DESIGN}

\section{A. Phase Shifter Design}

A reflection type phase shifter architecture with a 4 port branch line coupler and two reactive variable loads was selected due to the favorable tradeoff between loss, matching performance and compactness [13]. 16 phase shifter unit cells were fabricated in house on a Rogers TM4 substrate $\left(\varepsilon_{\mathrm{r}}=4.5\right)$ using a $35 \mu \mathrm{m}$ copper cladding process. Four ultracompact diode varactors [14] were soldered as in Fig. 3(a) to enable reactive load tuning. SMA connectors were also soldered for testing of individual phase shifters prior to integration into the phased array.

The change in active element impedance was evaluated for all scan angles of interest to ensure a return loss of $<-10 \mathrm{~dB}$ for $\pm 50^{\circ}$ in azimuth and elevation. Fig. 3(b) shows the unit cell return loss (for minimum capacitive loading) and phase shifter performance. The observed deviation from simulated performance was attributable to the low-tech fabrication process resulting in over-etching and modification of the optimum inductance values. Nonetheless, a $250^{\circ}$ phase tune range was achieved as shown in Fig. 3(c) which provided
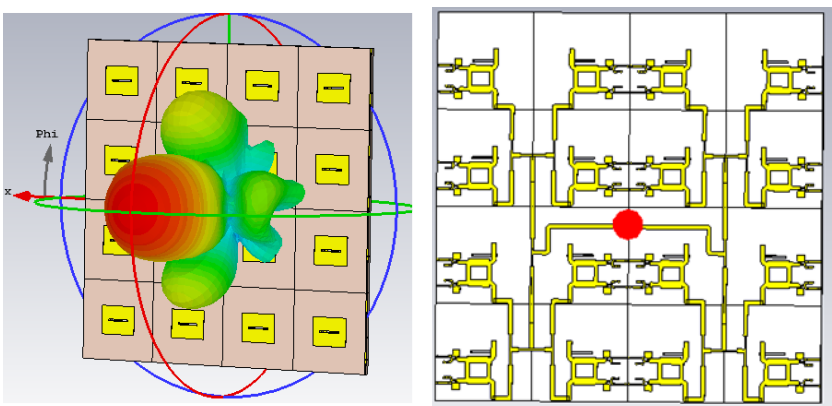

(a)

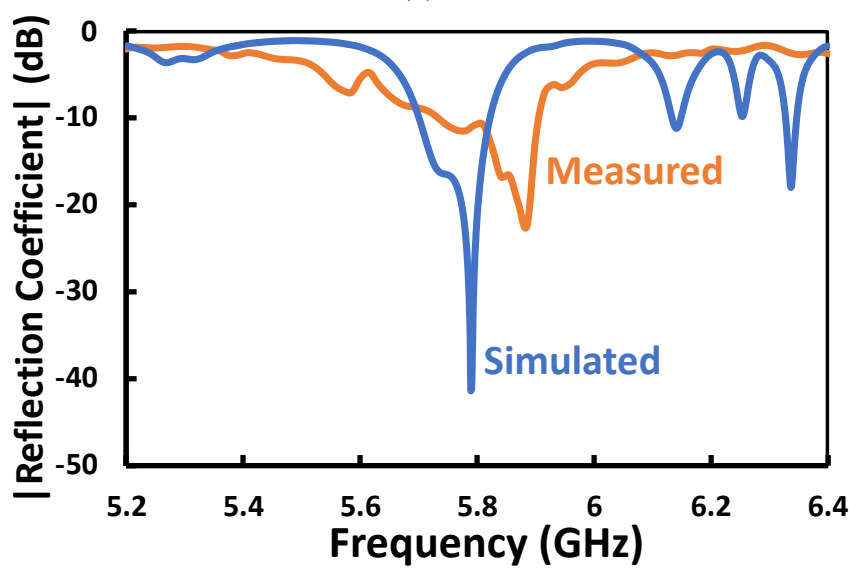

(b)

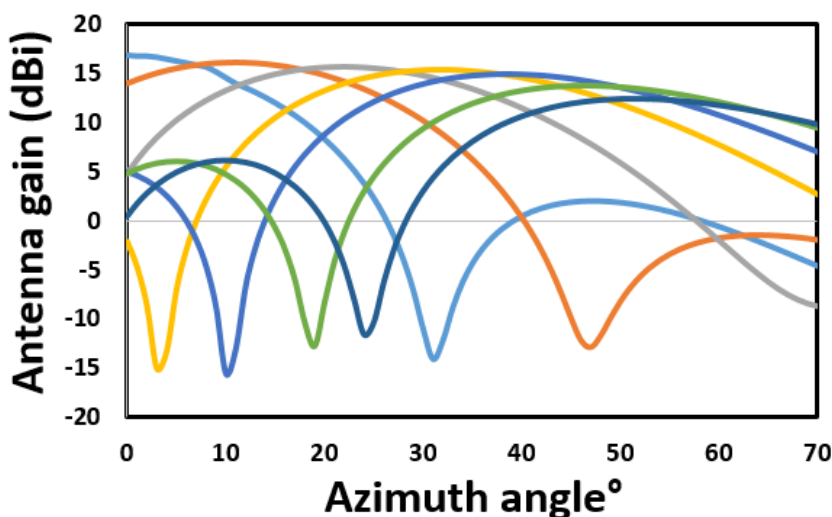

(c)

Fig. 4. (a) Phased antenna array and feeding network design. (b) Measured and simulated reflection coefficient for the phased antenna array at $5.8 \mathrm{GHz}$. (c) Simulated gain degradation due to antenna aperture change at high angles.

adequate phase shift for the beam scanning application.

\section{B. Antenna Array Design}

A 4x4-element microstrip antenna array was designed to achieve sufficient gain, scanning resolution and side lobe compression while ensuring low fabrication cost and encumbrance. The array was based on $5.8 \mathrm{GHz}$ resonating patch antenna laid on a multilayer stacked substrate to maximise gain, efficiency and bandwidth [15]. The bi-layer substrate (Rogers TMM4 antenna and Rogers TMM10i feed) supported a 14.42x $8.95 \mathrm{~mm}^{2}$ antenna patch. Every patch was fed via a $7 \times 0.6 \mathrm{~mm}^{2}$ slot resulting in $-10 \mathrm{~dB}$ return loss and better than $10 \%$ bandwidth across the targeted scan range $\left( \pm 50^{\circ}\right.$ in azimuth and elevation). A $1^{\circ}$ beam steering was calculated to require a $5^{\circ}$ phase shift [16] resulting in a scanning range of $\pm 72^{\circ}$ which is 


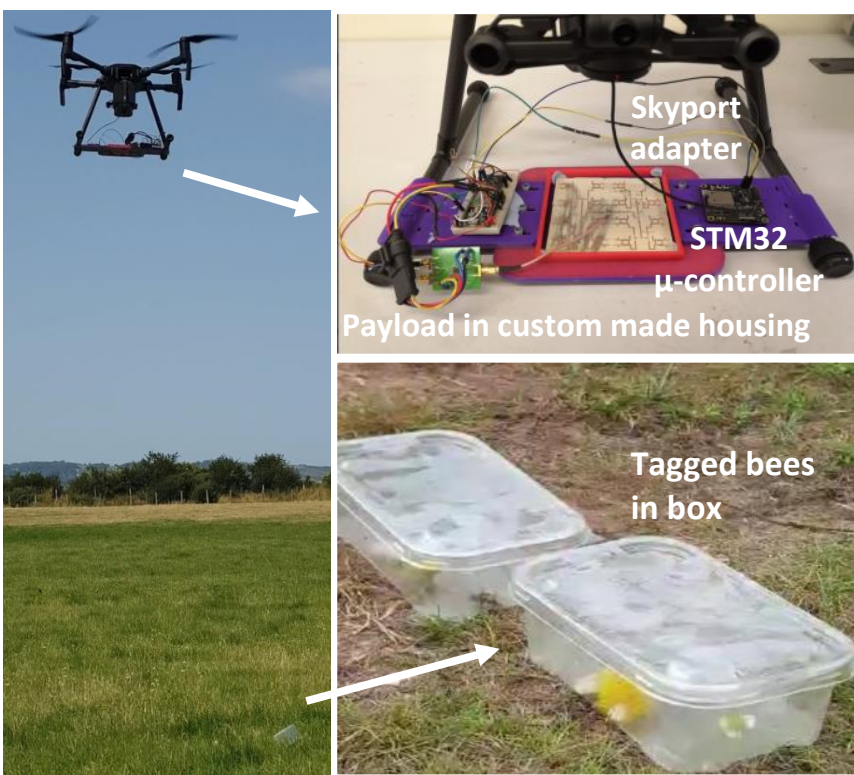

(a)

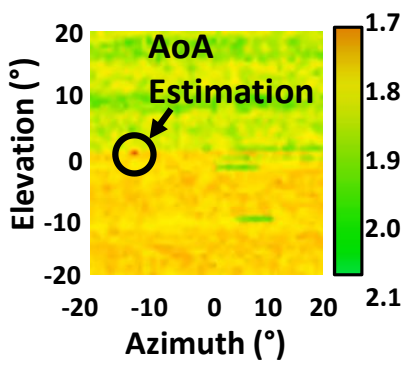

(b)

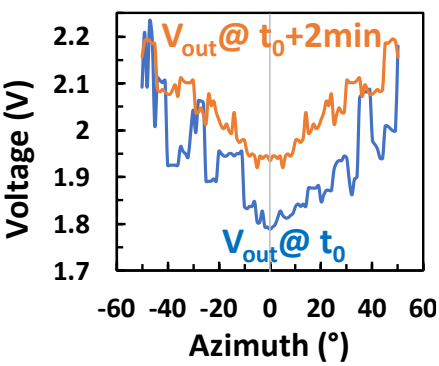

(c)
Fig. 5. (a) Tracking system operation in the field. Insets detail the receiver housing unit and connections along with tagged bumblebees in a box (discussed in VI). (b) Real-time heat maps outlining AOA estimate for the target's position and (c) RSSI time fluctuation for repeated measurements at same angle and distance.

reduced to $\pm 50^{\circ}$ by the phase shifter range. A $0.5 \lambda$ element spacing was enforced to prevent grating lobes at higher scan angles. The simulated antenna gain decay due to change in antenna aperture at higher scan angles ( 3dBi at 50 degrees) can be appreciated in Fig. 4(c).

For excitation of the $4 \times 4$ array from a single feeding point a Wilkinson power divider was designed. A high dielectric constant $\left(\varepsilon_{\mathrm{r}}=9.8\right)$ and low tangent loss $(\delta=0.002)$ Rogers TMM $10 \mathrm{i}$ substrate was selected to ensure a compact feeding network and high antenna efficiency, respectively. Via holes were manually drilled in the substrate for grounding of the varactor diodes soldered to each phase shifter, and the feed point was driven though a SMA connector.

Starting from design equations [16], multiple-parameter optimisation was afforded in CST studio [17] for the phased array to achieve return loss, gain and efficiency of $22 \mathrm{~dB}, 14.5$ $\mathrm{dBi}$ and $67 \%$, respectively, at $5.8 \mathrm{GHz}$. While the gain and efficiency performance could not be experimentally validated due to restricted lab access, the measured return loss is reported in Fig. 4(b). Deviation from the simulated performance was attributed to heterogeneity in the via holes size/alignment in addition to the afore mentioned fabrication tolerances.

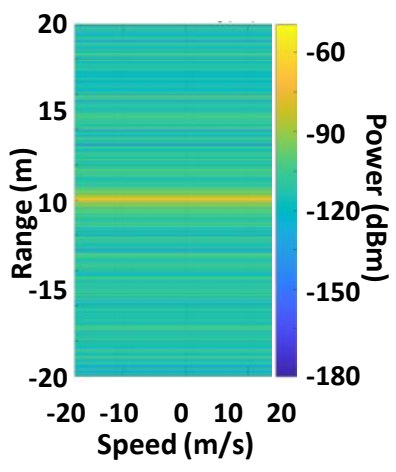

(a)

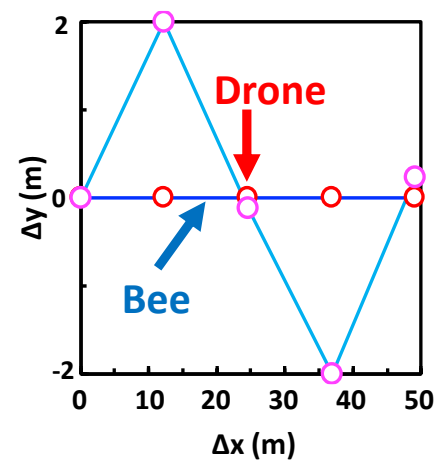

(b)

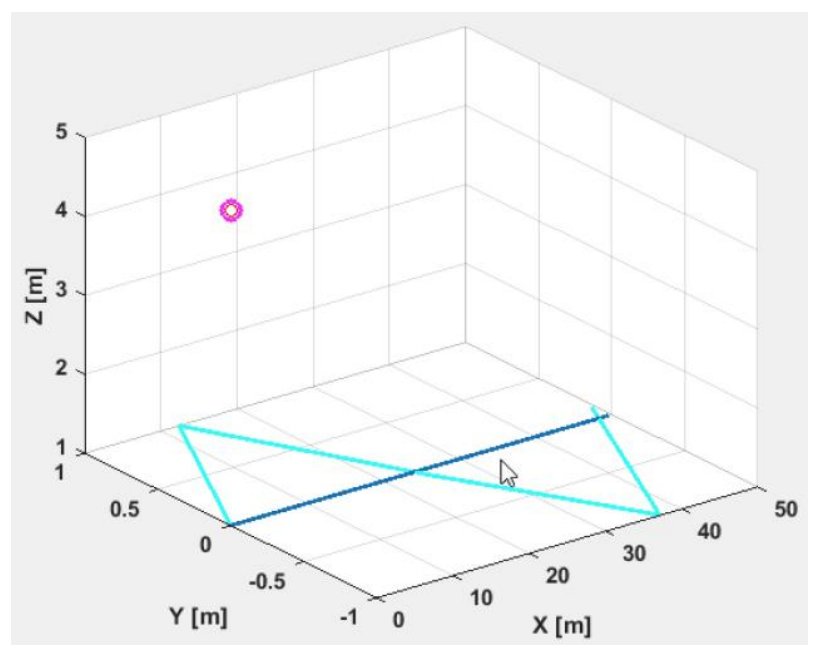

(c)

Fig. 6. (a) Simulation of Range-speed heat map from doppler analysis with a stationary receiver. (b) 2D projection of successful drone (symbols) tracking for linear and zigzag bee (solid curves) pathway at constant $12.3 \mathrm{~m} / \mathrm{s}$ speed. (c) 3D plot of unsuccessful drone tracking for linear and zigzag bee pathway at constant $12.4 \mathrm{~m} / \mathrm{s}$ speed resulting in drone hovering at initial point.

\section{DRONE SELF-PILOTING CONTROL}

The receiver system in III was capable of AOA detection within a $20 \mathrm{~m}$ range (see section $\mathrm{V}$ ) which was used both for bee localisation and to feed the automated tracking system. The DJI M200 drone [18] was chosen due to its $1 \mathrm{~kg}$ payload carrying capability, the possibility to integrate the receiver unit with the drone flight commands, and its 30 min battery duration. The use of DJIs software development kits (SDKs) (Payload SDK and Mobile SDK) permits the integration of the receiver system to the Matrice series of drones.

\section{A. AOA Readings}

A logarithmic detector [19] was used to transduce the received signal power into an output voltage. The voltage was fed into a processing unit where the received signal strength indicator (RSSI) was recorded for each scan angle of the beam. The RSSI was then condensed into heat maps similar to the one in Fig. 5(b) supporting real-time AOA estimation. As the input power into the device increased, the amplifiers sequentially moved into saturation resulting in signal clipping. Hence, the maximum received power AOA was inferred from the angle of 


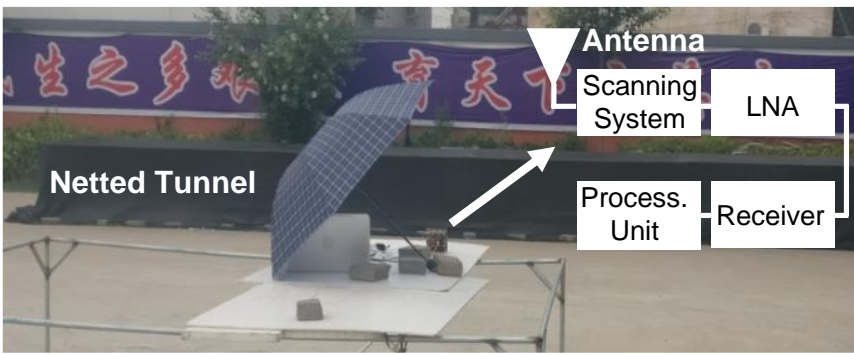

(a)

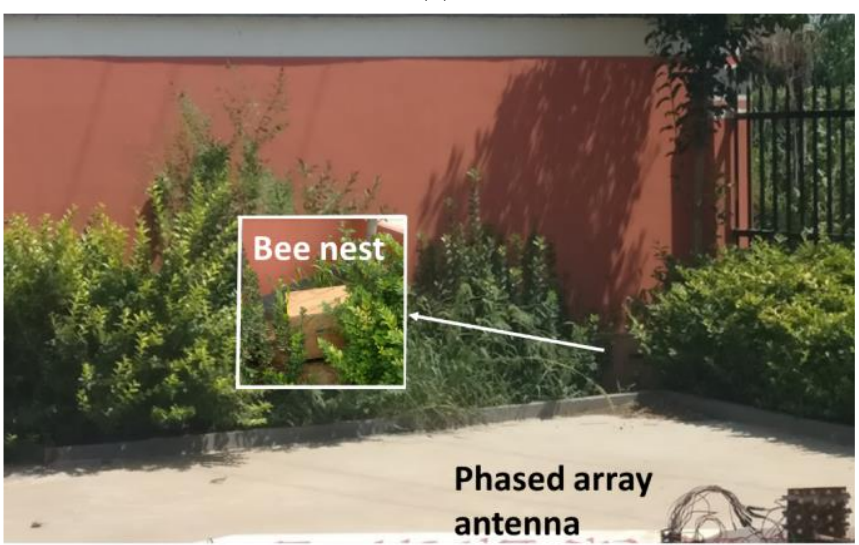

(b)

TABLE I

Foraging TIME AND DiRECTION OF FLIGHT LOG

\begin{tabular}{llllll}
\hline \hline \multicolumn{2}{l}{ Take off } & \multicolumn{3}{l}{ Landing } \\
\hline Time & Azimuth & Elevation & Time & Azimuth & Elevation \\
$0 \mathrm{~s}$ & $5^{\circ}$ & $5^{\circ}$ & $1425 \mathrm{~s}$ & $35^{\circ}$ & $30^{\circ}$ \\
$3 \mathrm{~s}$ & $-12^{\circ}$ & $17^{\circ}$ & $1428 \mathrm{~s}$ & $12^{\circ}$ & $10^{\circ}$ \\
$6 \mathrm{~s}$ & $25^{\circ}$ & $19^{\circ}$ & $1431 \mathrm{~s}$ & $-20^{\circ}$ & $22^{\circ}$ \\
$9 \mathrm{~s}$ & N/A & N/A & $1434 \mathrm{~s}$ & $-10^{\circ}$ & $5^{\circ}$ \\
\hline \hline
\end{tabular}

Fig. 7. Tests conducted with a stationary receiver releasing bumblebees (a) in a netted polytunnel and (b) near a nest. The receiver was placed $10 \mathrm{~m}$ away in both picture settings.

the read-out voltage minimum. Owing to the logarithmic detector $-70 \mathrm{dBm}$ sensitivity any AOA estimate with a RSSI > $2 \mathrm{~V}$, which corresponds to $\mathrm{P}<-70 \mathrm{dBm}$, was deemed to be erroneous and discarded. AOA and especially range estimation accuracy was also impaired by index fluctuations due to multipath reflection interference. Fig. 7(c) shows an example of the RSSI fluctuation over time for the same positional reading.

\section{B. Communication Protocol}

The inbuilt SDK enabled connection of our processing unit to the drone via serial Universal Asynchronous Receiver/ Transmitter (UART) communication, facilitating full integration of the receiver system and drone. The receiver was defined as the third-party payload and linked to the drone via a STM32F407VGTx microcontroller [20] enabling UART communication with the receiver system and Skyport connection via a ribbon cable visible in Fig. 1 and Fig. 5(a).

The UART link sent all captured data from the AOA estimation to the drone's processing unit at a $5 \mathrm{~Hz}$ rate. The data were stored, encrypted and transmitted to a remote base station through the Mobile SDK and a custom built application. This allowed us to take full advantage of the flight control

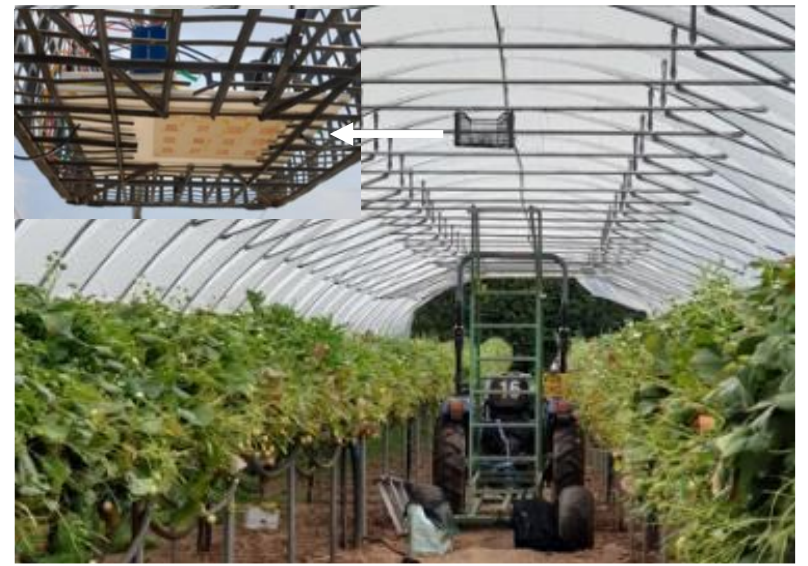

(a)
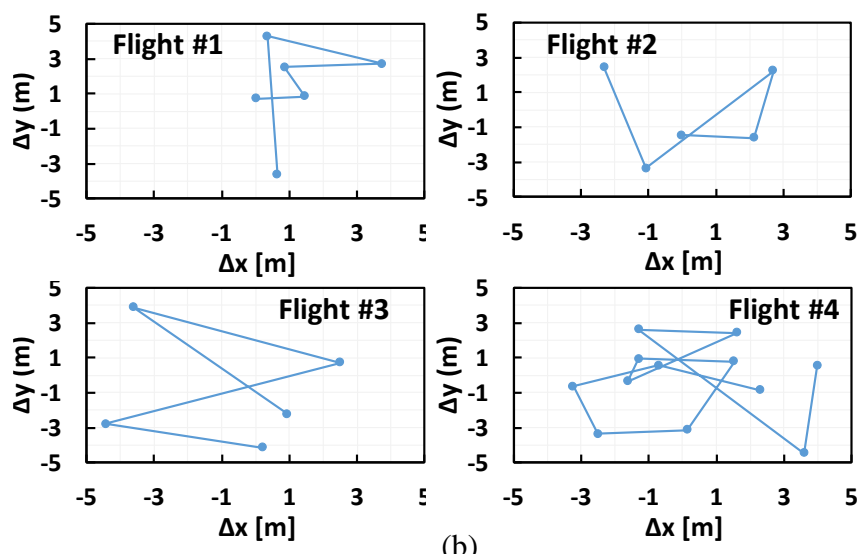

(b)

Fig. 8. (a) Tests conducted with a stationary receiver placed onto a polytunnel frame to detect flight of freely released bumblebees underneath strawberry plants. (b) Examples of recorded flight traces.

components in the Mobile SDK while also enabling lighter onboard processing. The AOA estimation was determined from decoding the incoming data of bee's bearing relative to the drone. Finally, the bearing was transmitted back to the drone as a command to update its position. While the tested system only allowed sharing of flight parameters to a PC based application upon completion of the track mission, future implementations could encompass environmental parameters and mapping of food (flower) source distributions. Statistical analysis could additionally enable automated discrimination of different activities, facilitating the type of tests reported in Fig. 1, along with prediction of foraging target and pollinator behavior.

\section{RANGE PREDICTION}

A simple model based on Friis equation was employed to determine the bee-UAV link budget both considering a stationary and a self-moving UAV scenario. The model was initially calibrated by combining the transmitting antenna in section III to a commercial receiving antenna [21], before validation with the phased array antenna. Properly attenuated continuous wave $(\mathrm{CW})$ sources rather than the tag transmitter were used for calibration consistency. Far field received power measurements were conducted both without a pre-amplifier $(\mathrm{r}=1 \mathrm{~m})$ and by adding a Low Noise Amplifier [22] before the 


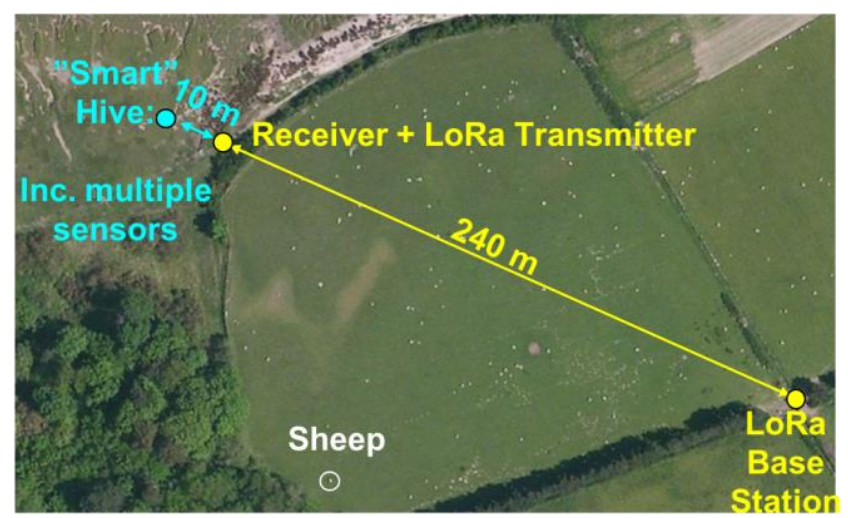

Fig. 9. LoRa link tested for validation on unobtrusive interrogation of the stationary receiver, which, in a future implementation could also include environmental parameter sensors.

logarithmic detector [19] ( $\mathrm{r}=2,10$, and $15 \mathrm{~m})$.

The maximum range for a stationary receiver was predicted using the calibrated model and with a conservative estimation of transmitter/receiver antenna gain $(1.7 / 14 \mathrm{dBi})$, transmitter/receiver loss $(1.0 / 1.0 \mathrm{~dB})$, additional loss (e.g. cross-polarisation: $3 \mathrm{~dB}$ ), LNA gain of $19 \mathrm{~dB}$ and noise figure of $2 \mathrm{~dB}$. Thus, the receiver sensitivity threshold was achieved for a distance of $18.9 \mathrm{~m}$. Doppler analysis was also performed yielding a frequency shift of $309 \mathrm{~Hz}$ for the bee maximum speed of $8 \mathrm{~m} / \mathrm{s}$ [23], which is well within the receiver bandwidth. Fig. 6(a) shows the range-speed response pattern of a moving target with a pre-set typical range of $10 \mathrm{~m}$. It can be seen that the target appears at the pre-set distance in spite of the minimal Doppler shift across the entire relative speed range.

The maximum range for a self-moving receiver was predicted both considering an ideal (0 s: Fig. 6(b)) and realistic (0.1,0.5, $1 \mathrm{~s}$ Fig. 6(c)) processing times with an AOA sampling period of $1 \mathrm{~s}$. A simple code was developed to mimic the drone being programmed to hover at the coordinates of the last point before reaching the receiver sensitivity limit. The maximum bee speed for successful detection was predicted to be $18.9 \mathrm{~m} / \mathrm{s}$, $16.8, \mathrm{~m} / \mathrm{s}, 12.3$ and $9.2 \mathrm{~m} / \mathrm{s}$ for $0 \mathrm{~s}, 0.1 \mathrm{~s}, 0.5 \mathrm{~s}$, and $1 \mathrm{~s}$ processing time, respectively, both for a linear and a zigzag bee pathway.

\section{EXPERIMENTAL}

The AOA detection system was tested by placing a $5.8 \mathrm{GHz}$ transmitter with comparable power level to the bee tags at known azimuth and elevation locations $10 \mathrm{~m}$ away from the stationary receiver. The beam was scanned every $1^{\circ}$ with the average of 3 RSSI readings prior to AOA estimation and resulted in a maximum system error of $\pm 5^{\circ}(\sim \pm 87 \mathrm{~cm})$. Detection was also achieved with the target $20 \mathrm{~m}$ away from the stationary receiver although saturation of the log-detector RSSI transducer [19] resulted in higher localization error.

For validation of the tracking system several experiments were conducted with bumblebees enjoying various freedom of flight degrees and using both a stationary and the fully automated drone receiver. For tag attachment to the bee's thorax the insect was temporarily immobilised and held in a sponge lined marking cage to attach the tag using removable glue.

\section{A. Bumblebees Tracking using a Stationary Receiver}

Bumblebees were initially released in a netted polytunnel to allow for bearing determination within the tested AOA detection range with low risk of tag loss. The receiver was placed at $10 \mathrm{~m}$ from the centre of the netted tunnel visible in Fig. 7(a) and the phased array antenna was set to scan every $1^{\circ}$ in azimuth and elevation. As any non-flight movements were unable to produce a detectable signal, position was only estimated during bee flight. Also, optically monitoring of the insect flight was impaired from the receiver and operator (10 $\mathrm{m}$ ) distance. Nevertheless, after loss of the $5.8 \mathrm{GHz}$ signal the operator was generally able to approach the tunnel and visually locate the stationary (e.g. grooming) insect position according to the last available bearing. While successful localisation statistics could not be drawn, this test empirically validated the $\sim 1 \mathrm{~m}$ resolution derived in the AOA test.

In the following tests the system was mounted on a static support $10 \mathrm{~m}$ from the nest, whilst tagged bees continuously left and returned to the nest visible in Fig. 7(b). A typical flight from and to the nest allowed recording of the azimuth/elevation coordinates, thus inferring flight duration (Tab. 1), along with the associated RSSI (not reported). It should be noticed that tagged bumblebees were able to roam within the nest unhindered by the tag, in contrast with harmonic radar telemetry [10] which requires tag removal before the bee can re-enter the nest.

The last test outlining the potential for a static receiver approach was conducted in a polytunnel environment with the setup portrayed in Fig. 8(a). A custom 3D housing unit was printed to contain the receiver unit and placed onto the polytunnel frame $5 \mathrm{~m}$ above the strawberry plants. The receiver was modified to include a SD card reader and a portable battery pack, which allowed continuous power supply and data collection for 24 hours. Example flight paths are displayed in Figure 8(b). While the tracking in this experiment was comfortably within the system detection range, the spatial resolution was affected by multipath effects from the metal structures underneath the flowers. Nevertheless, the present stationary receiver can already support monitoring/analysis of pollinators operating both in open air and greenhouse systems.

To further improve the ease of data acquisition, the stationary receiver was finally accessed through a Long Range (LoRa) board [24] hence showing the potential for: unobtrusive receiver interrogation from a $240 \mathrm{~m}$ range (Fig. 9); real-time data processing rather than periodical SD card access; integration of additional sensors (e.g. temperature, humidity and lighting) for multi-parameter monitoring of colony health.

\section{B. Bumblebees Tracking Using a Self-Moving Receiver}

Tagged bumblebees were initially released in the plastic, semitransparent container $25 \times 15 \times 10 \mathrm{~cm}^{3}$ in Fig. 5(a). The drone hovered at $5 \mathrm{~m}$ altitude at several locations with horizontal projection $<20 \mathrm{~m}$ from the tagged bee. When the bee flapped its wings inside the box the system was able to locate the target and autonomously move towards its location. The drone autonomously updated its bearing and the internal log readings were subsequently plotted for two representative experiments 


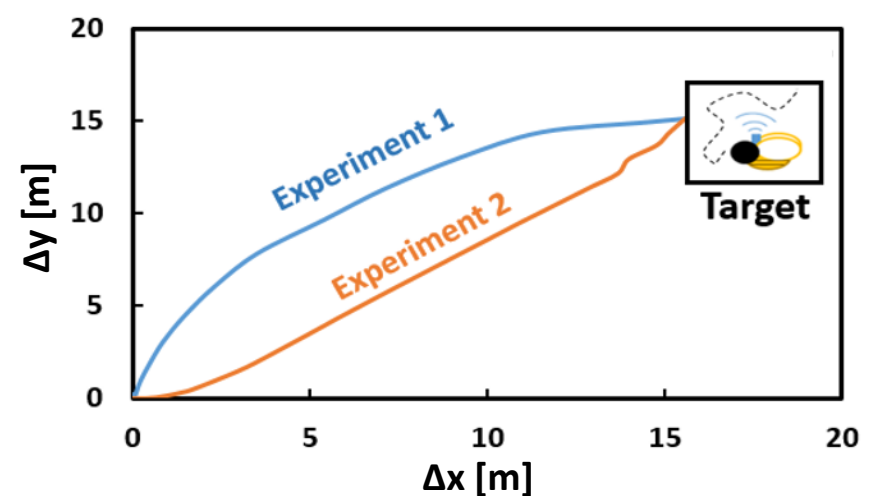

(a)

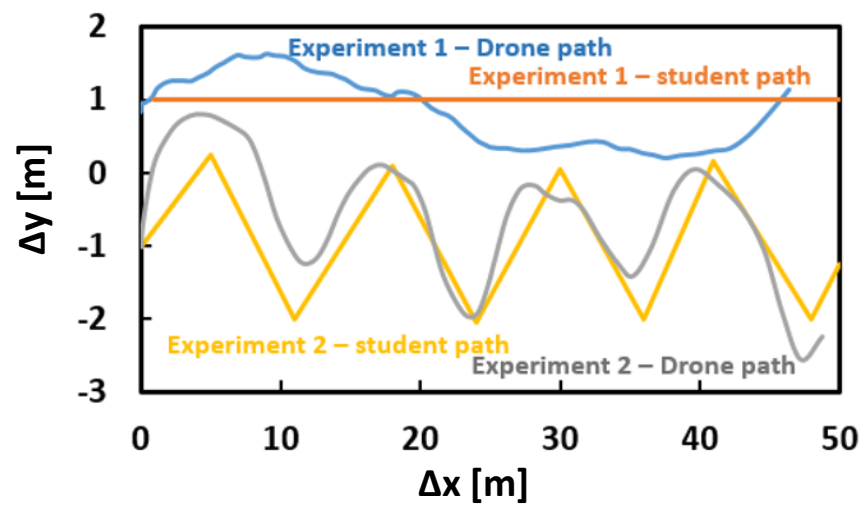

(b)

Fig. 10. (a) Update in drone's position over time while tracking (a) tagged bumblebees flying in a plastic container; (b) Student walking along different paths with a handheld transmitter.

(Fig. 10). The results in Fig.10(a) match the prediction in Fig. 6(a) while demonstrating increased range, speed and adaptive control over a previous automated tracking test in [7].

Further tests were conducted to demonstrate the long-range autonomous tracking potential of unconstrained targets. Tracking of freely moving tags (carried by a student assistant) were achieved over a $50 \mathrm{~m}$ distance with battery-powered and a handheld Voltage Controlled Oscillator (VCO) emitting a Continuous Wave (CW) $3.6 \mu \mathrm{W} 5.8 \mathrm{GHz}$ signal. As shown by Fig. 10(b) the drone's flight path followed the tag emitter and recorded both linear and a zig zag trajectory with an acceptable margin of error $\left( \pm 5^{\circ}\right)$. The margin of error can be explained by the drone being programmed to simultaneously update its roll, pitch and yaw, generating a deviation between the target and drone's path.

Whilst the preliminary tests were limited to a $50 \mathrm{~m}$ distance and up to $25 \%$ of typical bee flight speed, the results in Fig. 10(b) match the detection range prediction in Fig. 6(b). Hence, our results suggest that tracking over a substantially increased distance could be achieved provided a proper tradeoff between pulsing frequency and processing time is ensured.

\section{DISCUSSION}

Development of the present telemetry system required an inherently multi-disciplinary approach and several approximations were applied to tackle complex problems. In some cases, a more rigorous analysis, or a more general solution to the encountered challenges seem possible and set a pathway towards systemic improvement.

On the energy harvesting side: the fact that the tag represents a considerable weight portion of the bee+harvester system, and that the insect's thorax is not a rigid frame system are bound to require a more advanced (2-body problem) formulation [25]. As such, it is possible that the maximum power output from bee vibration was not achieved, which potentially presents an opportunity for increased communication range.

Range could be further increased through the adoption of -85 $\mathrm{dBm}$ sensitivity receivers [26], higher performance LNAs [27], and commercially manufactured antennas. Indeed, a second generation phased array has already been developed and commercially fabricated but could not be tested during restricted lab access.

Similarly, higher tuning range could be achieved through phase shifter design/fabrication enhancement and potentially enable increased beam scanning range. However, it remains to be seen if such endeavor could overcome radiation pattern degradation due to array elements coupling and antenna aperture decrease at high scan angles [15].

For stationary receiver settings with moderate range $(<5 \mathrm{~m})$, signal scattering from unavoidable reflectors, as the metallic polytunnel frame, can limit monitoring resolution. In similar settings CW Doppler detection [28] is now being investigated to avoid masking signals from stationary reflectors.

The presented model for range prediction intentionally oversimplifies the drone operation by assuming it instantaneously achieves the target waypoint once the processing time has expired. This is partially justified by the drone maximum speed $(17 \mathrm{~m} / \mathrm{s})$ being twice as high as the bee's maximum speed. However, the question of what speed would ensure highest tracking success (for example avoiding overshooting etc.) is clearly an open one and could not be extensively investigated. It is expected that adaptive speed and roll control would be instrumental in tracking of abrupt speed/direction changes of nimble pollinators.

Nevertheless, the combination of modeling and experimental work presented here provided key insights into the system detection range and spatial resolution. In spite of the limited $(\sim 1 \mathrm{~m})$ resolution the present system shows significant promise for investigation of how pollinators operate in space. For

TABLE II

POLLINATOR TRACKING SYSTEM COMPARISON

\begin{tabular}{cccccc}
\hline Reference & $\begin{array}{c}\text { Technique and } \\
\text { Frequency [Hz] }\end{array}$ & Tag Type and Weight [mg] & Required Terrain & $\begin{array}{c}\text { Detection Range } \\
\text { Order [m] }\end{array}$ & System cost [\$] \\
\hline$[4][10]$ & Harm. Radar: $9.4-18.8 \mathrm{G}$ & Passive/Active: $\sim 4-200$ & Flat and open & 1000 & $>.1$ \\
{$[29]$} & RFID: $13.6 \mathrm{M}$ & Passive: $\sim 10$ & All & $10 \mathrm{k}$ \\
This work & RSSI: $5.8 \mathrm{G}$ & Active: $30-80$ & All & 10 & $<1 \mathrm{k}$ \\
\hline \hline
\end{tabular}


example, it could be already used to assess if the recorded flight direction matched the known location of food supplies and assess number of trips a bee takes outside the nest each day.

A comparison of the proposed system performance to stateof-the-art pollinators tracking systems is presented in Table 2 . Longer range can be achieved through radar approaches whilst RFID technology [29] relies on inherently lighter tags. Yet, the competitive range/weight tradeoff obtained in this work shows considerable promise for tracking of pollinators as small as $A$. mellifera. The automated tracking capacity in this technology potentially delivers a uniquely versatile and affordable solution - with set-up and maintenance cost mainly dictated by the UAV system - for insect tracking in rough terrain.

\section{CONCLUSION}

A $5.8 \mathrm{GHz}$ telemetry system was herein presented along with a simple range prediction model to empower tracking and monitoring of a variety of bee species. The system consists of a battery-less transmitter, a phased array antenna, and a selfpiloting drone acting on the received RSSI signal while harvesting real time localisation data. Tests with a stationary receiver demonstrated the capability for unobtrusive pollinator monitoring and foraging time estimation both near hive (outdoor) and in a polytunnel system. Modeling work and tests with a self-piloted drone suggested that tracking of targets flying up to $8 \mathrm{~m} / \mathrm{s}$ is possible as long as bearing estimation and drone processing time are kept within $1 \mathrm{~s}$.

\section{REFERENCES}

[1] R. Winfree, I. Bartomeus and D. Cariveau, "Native pollinators in anthropogenic habitats", Annи. Rev. Ecol. Evol. Syst., vol. 42, no. 1, pp. 1-22, Nov. 2011. Accessed on: June, 2020, DOI: 10.1146/annurev-ecolsys-102710-145042, [Online].

[2] J. C. Makinson, et. al., "Harmonic radar tracking reveals random dispersal pattern of bumblebee (Bombus terrestris) queens after hibernation", Sci. Rep., vol. 9, no. 1, pp. 1-11, Nov. 2019. Accessed on: June, 2020, DOI: 10.1038/s41598019-40355-6, [Online].

[3] J. L. Woodgate, J. C. Makinson, K. S. Lim, A. M. Reynolds and L. Chittka, "Life-long radar tracking of bumblebees", PLOS ONE, vol. 11, no. 8, pp. e0160333, Aug. 2016. Accessed on: July, 2020, DOI: 10.1371/journal.pone.0160333, [Online].

[4] M. Lihoreau, et al., "Radar tracking and motion-sensitive cameras on flowers reveal the development of pollinator multi-destination routes over large spatial scales". PLoS Biol., vol 10, no. 9, pp. e1001392, Sept. 2012, Accessed on: July, 2020 DOI: 10.1371/journal.pbio.1001392, [Online].

[5] M. A. Hall, J. Jones, M. Rocchetti, D. Wright and R. Rader, "Bee visitation and fruit quality in berries under protected cropping vary along the length of polytunnels", J. Econ. Entomol., vol. 113, no. 3, pp. 1337-1346, June, 2020. Accessed on: July, 2020, DOI: 10.1093/jee/toaa037, [Online].

[6] J.M. Mola, and N.M. Williams. "A review of methods for the study of bumble bee movement." Apidologie: no. 50 pp. 497514. Jan. 2019, DOI: 10.1007/s13592-019-00662-3, [Online].

[7] J. Shearwood, D. M. Hung, P. Cross, S. Preston and C. Palego, "Honeybee localization using an energy harvesting device and power based angle of arrival estimation" in IEEE MTTS Int. Microw. Symp., Philadelphia, PA, USA, June 1015, 2018, pp. 957-960.
[8] J. Shearwood, et al., "Localization and tracking bees using a battery-less transmitter and an autonomous unmanned aerial vehicle" in IEEE MTTS Int. Microw. Symp., Los Angeles, CA, USA, June 21-26, 2020, pp. 1263-1266.

[9] E. Aktakka, H. Kim and K. Najafi, "Energy scavenging from insect flight", J. Micromech. Microeng, vol. 21, no.9, pp. 095016, 2011. Accessed on: June, 2020, [Online].

[10] M. Hagen, M. Wikelski, W.D. Kissling, "Space use of bumblebees (Bombus spp.) revealed by radio-tracking", PLOS ONE, vol. 6, no. 5, pp. e19997, 2011. Accessed on: June, 2020, DOI: 10.1371/journal.pone.0019997, [Online].

[11] "Piezoelectric Energy Harvesters," Piezo.com, 2020, [Online], Available: https://piezo.com. Accessed on: July 2020.

[12] "PMEG4002EL data sheet." [Online], Available: https://docs.rs-online.com/a49e/0900766b80b367e2.pdf Accessed on: July 2020.

[13] C. Cheng, C. Ko, A. Morris and G. M. Rebeiz, "A very low loss 1.9-2.1 GHz RF MEMS phase shifter," in IEEE MTTS Int. Microw. Symp., Montreal, QC, Canada, 2012, pp. 1-3.

[14] Macom, "MA46H120 GaAs Constant Gamma Flip-Chip Varactor diode." [Online], Available: https://cdn.macom.com/datasheets/MA46H120\%20Series.p df Accessed on: June, 2020

[15] B. L. Ooi, S. Qin and M. S. Leong, "Novel design of broadband stacked patch antenna", IEEE Trans. Antennas Propag., vol. 50, no. 10, pp. 1391-1395, 2002. Available: 10.1109/tap.2002.802087, [Online].

[16] P. W. Hannan, "The element-gain paradox for a phased-array antenna," IEEE Trans. Antennas Propag., vol. 12, no. 4, pp. 423-433, July, 1964. Accessed on: July, 2020, DOI: 10.1109/TAP.1964.1138237, [Online].

[17] Studio Suite, Dassault Systems, Vélizy-Villacoublay, France 2020.

[18] "Matrice 200 Series." [Online]. Available: https://www.dji.com/uk/matrice-200-series

[19] "HMC602LP4 Data Sheet," Analog Devices, Inc., [Online]. Available: https://www.analog.com/media/en/technicaldocumentation/data-sheets/hmc602.pdf

[20] "STM32F4DISCOVERY," [Online]. Available: https://www.st.com/en/evaluationtools/stm32f4discovery.html

[21] "B07MHNVYVQ $5.8 \mathrm{GHz} 3 \mathrm{dBi}$ Omnidirectional Antenna" [Online] Available: https://www.amazon.co.uk/s?k=B07MHNVYVQ\&ref=404 _search

[22] "HMC902LP3E Data Sheet," Analog Devices, Inc. [Online]. Available: https://www.analog.com/media/en/technicaldocumentation/data-sheets/hmc902lp3e.pdf

[23] A. M. Wenner, "The flight speed of honeybees: a quantitative approach," J. Apic. Res., vol. 2, no. 1, pp. 25-32, 1963. Accessed on: July, 2020, DOI: 10.1080/00218839.1963.11100053, [Online].

[24] “RAK811 LoRa / LoRaWAN module.” [Online]. Available: https://uk.pi-supply.com/products/rak811-lora-lorawanmodule

[25] Roundy, P. K. Wright and J. Rabaey, "A study of low level vibrations as a power source for wireless sensor nodes", Comput. Commun., vol. 26, no. 11, pp. 1131-1144, 2003. Accessed on: July, 2020, DOI: 10.1016/s01403664(02)00248-7, [Online].

[26] "AWM662F RX," Airwave Tech. Inc. [Online]. Available: https://www.airwave.com.tw/en/product-441206/5-8GHzRF-Modules-AWM662F-RX.html

[27] "HMC902 Data Sheet," Analog Devices, Inc. [Online]. Available: https://www.analog.com/media/en/technicaldocumentation/data-sheets/hmc902-die.pdf

[28] H. Aumann, B. Payal, N. W. Emanetoglu and F. Drummond, "An index for assessing the foraging activities of honeybees with a doppler sensor," in IEEE Sensors Appl. Symp., Glassboro, NJ, USA, 2017, pp. 1-5.

[29] P. Nunes-Silva et al., "Applications of RFID technology on the study of bees". Insectes Sociaux, vol 66, no.1, pp.15-24. Sept.2018 Accessed on: July, 2020, [Online]. 


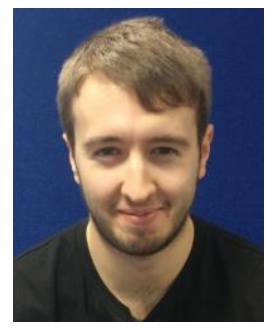

Jake Sherwood received the BEng degree in electronic engineering from Bangor University, UK, in 2016 where he has also completed his $\mathrm{PhD}$ degree in electronic engineering, in 2020. He has been a research scientist at Bangor University from 2020. His research interests include the development of telemetry systems for tracking and monitoring Insects, energy harvesting, antenna arrays and biomedical research.

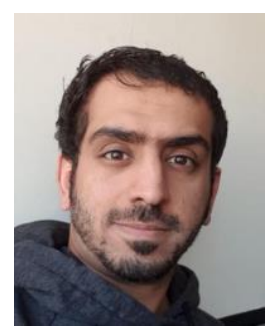

Nawaf Aldabashi received the BEng degree in Computer Systems Engineering and the MSc degree in Electronic Engineering from Bangor University in 2013 and 2016 respectively, where he is currently pursuing his Ph.D. degree with the department of Electronic Engineering. His research interests are the design, integration and application of continuous wave and frequency modulated continuous wave radar in addition to nanotechnology.

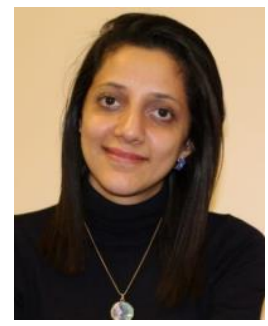

Amira Eltokhy received the M.S. degree in Wireless Mobile Communication systems, in 2015, and the Ph.D. degree in Microwave Engineering, in 2019, from the University of Greenwich, UK. She has been a postdoctoral associate at Bangor University, UK in 2019-2020. Her research interests include microwave systems and Biomedical Engineering in addition to Artificial Intelligence integration in both fields.

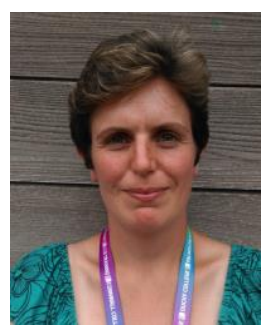

Elizabeth L. Franklin is a lecturer and degree programme manager at Cornwall College Newquay since 2019. She gained her degree in Environmental Biology from Plymouth University followed by a $\mathrm{PhD}$ at the University of Bristol studying animal behaviour. She has taught and been active in research both nationally and over a 2 year of postdoctoral fellowship at the University of Guelph, Canada. She is an entomologist and her research involve the study of the behaviour ants and bumble bees.

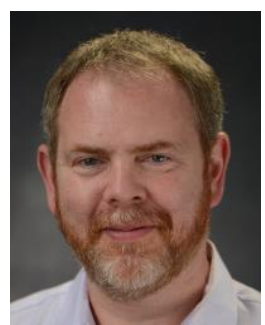

Nigel E. Raine is the Rebanks Family Chair in Pollinator Conservation, Professor at the University of Guelph. He is a global leader in the fields of behaviour, ecology and conservation of pollinators, and has spent much of the last decade working on the impacts of environmental stressors (such as pesticide exposure) on wild bees. Raine and his team use a range of technologies including RFID tagging, radar tracking and radio-telemetry to monitor the behaviour of bees and butterflies in the laboratory and field.

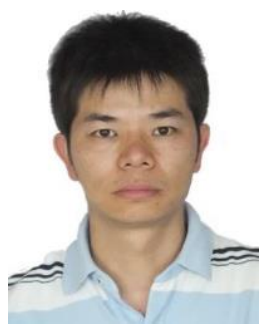

Chaochun Zhang's received the B. Sc. Degree from the Southwest University, China, 1996 and both M. Sc. (2002) and $\mathrm{PhD}$. (2010) degrees from China Agricultural University, where he is now an Associate Professor at the Department of Plant Nutrition. His research areas encompass the interspecific interactions and nutrient integrated management of intercropping systems.

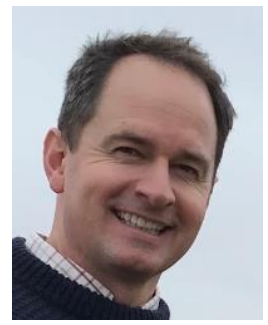

Edward Palmer is Group Technical Director and Business Development Manager and also leads the R\&D Team within S\&A Produce. He has extensive experience in all areas of agriculture having driven market outperformance with leading global retailers. $\mathrm{He}$ is now pioneering industry leading projects around robotics and sustainable plant based packaging solutions. Ed is a key advocate for new tracking technology that allow bees to behave as naturally as possible while promoting investigation of human impact and cohabitation strategies.

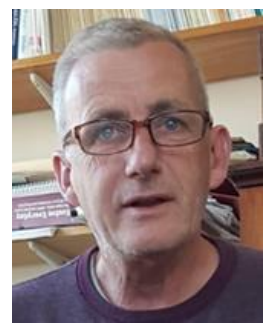

Paul Cross is Lecturer in the Environment at Bangor University, UK. His research portfolio encompasses an Agri-tech in China: Newton Network+ project to evaluate a UAV bee tracking system; the use of honey-bee morphometrics to determine racial strains of ecotypes in the UK involving collaboration with the National Bee Centre Wales; the impact of neonicotinoids on solitary bees; assessing racial purity in UK honey bees; bee dispersal rates and space use; and evaluating the potential of beekeeping to alleviate poverty in sub-Saharan Africa.

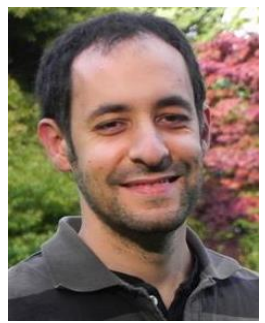

Cristiano Palego received the MEng. Degree in Electronics from the University of Perugia, Italy, in 2003, and the Ph.D. degree in Microwaves and Optoelectronics from the University of Limoges, France, in 2007. He was a research scientist at Lehigh University, USA (2007-2017). In 2013 he joined Bangor University, UK as an associate professor and in smart sensors and instrumentation. His research interests include bioelectronics, minimally invasive sensors and energy harvesting along with multitechnology integration and reconfigurable antenna arrays. 